\title{
The Cluster Analysis of Pulsar Parameters
}

\author{
Igor' F. Malov, Oleg I. Malov \\ Astro Space Center, Lebedev Physics Institute, Moscow, Russia
}

We have used the catalogue of (Taylor et al., 1993) to investigate the problem of a classification of pulsars. Five samples have been analysed by the main components method and by methods of the cluster analysis.

i) 15 pulsars with 14 known parameters $P, \dot{P}, W_{50}, L$ (Malov et al., 1994), $B$, $z$,spectral index $a$, frequencies of maximum $\nu_{M}$ and minimum $\nu_{c}$ in a spectrum (Malofeev et al., 1994), an angle between magnetic moment and rotation axis $\beta$ (Malov, 1994), the transformation coefficient $\eta=L / \dot{E}$, magnetic field near the light cylinder $B_{l c}$ and a maximum derivative of a position angle $C=(d \psi / d \phi)_{\max }$ (Malov, 1990).

ii) 130 objects with 10 parameters (the spectral characteristics $\alpha, \nu_{M}, \nu_{c}$ and the parameter $C$ were excluded from the consideration).

iii) 218 pulsars with 4 known parameters $P, \dot{P}, L$ and $z$. These parameters can be considered as independent variables.

iv) 89 sources with the same 4 parameters. This sample consists of two subsamples: 65 pulsars with $P>1 \mathrm{~s}$ and 24 ones with $P<0.1 \mathrm{~s}$.

v) 24 pulsars with $P<0.1 \mathrm{~s}$ from the previous sample.

\section{Conclusions}

1. Pulsars with long periods $(P>1 \mathrm{~s})$ belong apparently to a single population described by a single model and by one emission mechanism. They differ one from another by their geometry and by initial values of $P$ and $\dot{P}$. These parameters determine scales and a structure of magnetospheres, electric and magnetic fields, transformation coefficients etc.

2. It is shown that pulsars with $P<0.1 \mathrm{~s}$ and with $P>1 \mathrm{~s}$ form two different groups in the space of 4 parameters $P, \dot{P}, L, z$.

The distribution of 89 objects (the sample iv) on the plane of two main components:

$$
\begin{aligned}
& F_{1}=0.053 \log z-0.416 \log P-0.883 \log \dot{P}+0.208 \log L \\
& F_{2}=0.210 \log z-0.199 \log P-0.296 \log \dot{P}-0.910 \log L
\end{aligned}
$$

shows that pulsars with short periods and long-periodic ones are two unintersecting populations.

This'result confirms the hypothesis on two types of pulsars (Malov and Suleymanova, 1982) with different emission mechanisms.

3. Pulsars with short periods divide into two groups: i) young pulsars similar to PSR $0531+21$ and PSR 0833-45 and ii) recycled pulsars on the plane 
of two main components:

$$
\begin{aligned}
& F_{1}{ }^{*}=0.088 \log z-0.169 \log P-0.961 \log P-0.201 \log L \\
& F_{2}{ }^{*}=0.451 \log z+0.168 \log P+0.191 \log P-0.856 \log L
\end{aligned}
$$

These two groups form two left maxima in the histogram $N(P)$.

Recycled pulsars turn never into Crab-like pulsars. In fact if $\dot{P}_{-15} \leq 10^{-3}$ it is necessary more than $10^{9}$ years to go from $P=5 \mathrm{~ms}$ to $P=40 \mathrm{~ms}$. So all three maxima in the histogram $N(P)$ are apparently real.

\section{References}

Malov, I.F., Malofeev, V.M., and Sen'yo, D.S. 1994, Astron. Zh., 71, 762 Malofeev, V.M. et al. 1994, Astron. Astrophys., 285, 201

Malov, I.F. 1990, Astron. Zh., 67, 377

Malov, I.F., Suleymanova, S.A. 1982, Astrofizika, 18, 107

Taylor, J.H., Manchester, R.N., and Lyne, A.G. 1993, Astrophys. J. Suppl Ser., 88,529 